\title{
Image Reconstruction from Amplitude-Only and Phase-Only Data in the Fractional Fourier Domain ${ }^{1}$
}

\author{
T. Alieva and M. L. Calvo \\ Universidad Complutense de Madrid, Facultad de Ciencias Físicas, Departamento de Óptica, Madrid, 28400 Spain \\ e-mail: talieva@fis.ucm.es; mlcalvo@fis.ucm.es \\ Received December 20, 2002
}

\begin{abstract}
Image reconstruction from amplitude-only and phase-only data in the fractional Fourier domain applying the inverse fractional Fourier transform is analyzed on the examples of perfect edges of different contrasts and real-world image. (C) 2003 MAIK "Nauka/Interperiodica”.
\end{abstract}

The question of the importance of phase and amplitude in the Fourier domain has been studied in many publications [1-4]. There is a widespread belief that the Fourier phase is more important than the Fourier amplitude, based on the fact that the main characteristics of natural images can be recovered from phase-only data by applying the inverse Fourier transform (FT). Indeed, the Fourier amplitude of natural images is mostly concentrated in the low-frequency region. By eliminating the amplitude information, we stress the high frequencies of the Fourier spectrum. This produces an edgeenhancement effect. Nevertheless, it was shown in [4] that the dominance of Fourier phase or amplitude strongly depends on the symmetry and reality of the input signals.

Nowadays, the fractional FT, recently introduced in optics [5-6], plays an important role in optical signal and image processing. Convolution and correlation operations in the fractional Fourier domain are applied for pattern recognition, noise reduction, watermarking, encryption, and so on $[5,7]$. Often, the application of phase-only filters (POF) in the fractional Fourier domain is proposed for these tasks. Nevertheless, the effect of POFs in fractional Fourier domains has not been discussed in detail at present.

In this paper, we consider the possibility of image reconstruction from amplitude-only or phase-only data in fractional Fourier domains on the examples of perfect edges of different contrasts and natural real image.

The fractional FT [5] of $f(x)$ is a generalization of the ordinary FT

$$
\begin{gathered}
F_{\alpha}(u)=R^{\alpha}[f(x)](u)=A_{\alpha}(u) \exp \left(i \varphi_{\alpha}(u)\right) \\
=\int f(x) \frac{\exp (i \alpha / 2)}{\sqrt{i \sin \alpha}} \exp \left(i \pi \frac{\left(x^{2}+u^{2}\right) \cos \alpha-2 x u}{\sin \alpha}\right) d x .
\end{gathered}
$$

\footnotetext{
${ }^{1}$ This article was submitted by the authors in English.
}

Its kernel depends on the parameter $\alpha$, which can be interpreted as a rotation angle in phase space. Thus, for $\alpha=0$, the kernel corresponds to the identity transform; for $\alpha=\pi / 2$ and $\alpha=3 \pi / 2$, it reduces to the FT and the inverse FT, respectively. The inverse transform to the fractional FT for parameter $\alpha$ is the fractional FT for the parameter $-\alpha: R^{-\alpha}\left[F_{\alpha}(u)\right](x)=f(x)$.

The fractional FT describes, in the paraxial approximation of the scalar diffraction theory, optical beam propagation through a medium with a quadratic refractive index. The fractional FT of a complex field amplitude $f(x)$ can be performed by using a thin lens with focal distance $f$, with the input and output planes located at distances $d=2 f \sin ^{2} \alpha / 2$ before and after the lens, respectively [6].

The question of the importance of the phase $\varphi_{\alpha}$ or the amplitude $A_{\alpha}$ in a certain transform domain is usually related to the possibility of signal reconstruction from only the phase or only the amplitude information by applying the inverse transform. Thus, we will study the operations $R^{-\alpha}\left[A_{\alpha}(u)\right](x)$ and $R^{-\alpha}\left[\exp \left(i \varphi_{\alpha}(u)\right)\right](x)$, regarding them as signal reconstruction from amplitude-only and phase-only data in the fractional Fourier domain. For $\alpha=\pi / 2$, one gets the signal reconstructed from the amplitude and the phase of the Fourier transform of the original signal, respectively. Because we are interested in the reconstruction of real and positive images, the amplitudes $\left|R^{-\alpha}\left[A_{\alpha}(u)\right](x)\right|$ and $\left|R^{-\alpha}\left[\exp \left(i \varphi_{\alpha}(u)\right)\right](x)\right|$ will be analyzed. It is easy to see that, if a signal is shifted from the coordinate origin, which in a real optical realization of the fractional FT corresponds to the optical axis, by $s$, then the signal reconstructed from the amplitude-only information in the fractional $\alpha$ domain through the procedure $\left|R^{-\alpha}\left[A_{\alpha}(u)\right](x)\right|$ will be shifted by $s \cos ^{2} \alpha$. In the meantime, the signal reconstructed from the phase information is shift-covariant.

First, we consider the reconstruction of the perfect edges from the amplitude-only and phase-only infor- 
(a)

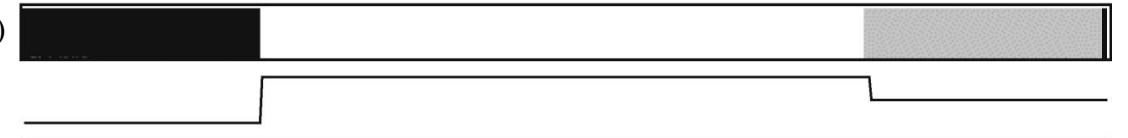

(b)

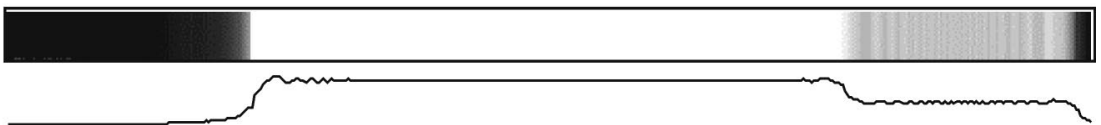

(c)

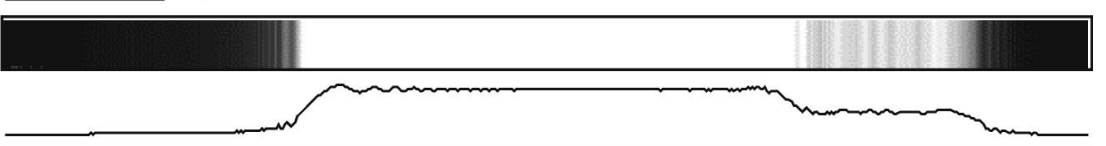

(d)

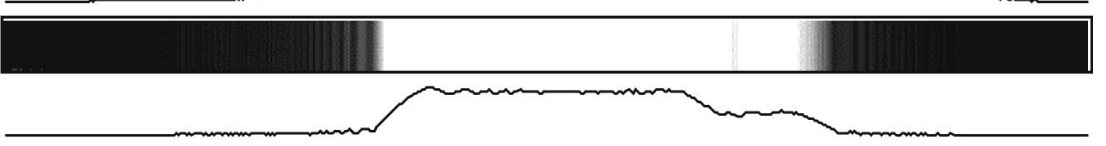

(e)

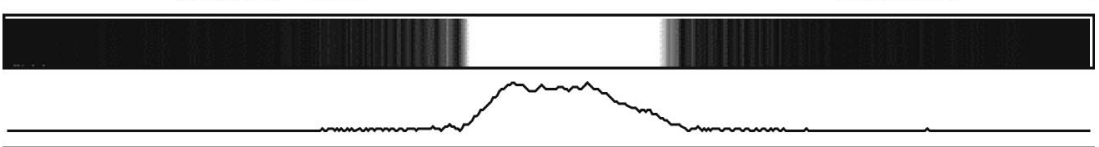

(f)

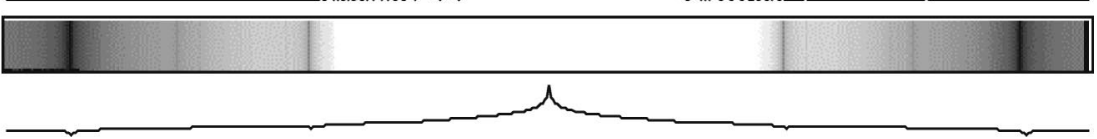

Fig. 1. Reconstruction of perfect edges of different contrasts $(C=1$, left side; $C=0.3$, right side) from the amplitude of the fractional FT for different $\alpha$ : (a) original image and (below) its line profile; (b-f) image reconstructed from the amplitude of the fractional FT for $\alpha=(b) \pi / 20$, (c) $3 \pi / 20$, (d) $\pi / 4$, (e) $7 \pi / 20$, and (f) $\pi / 2$.

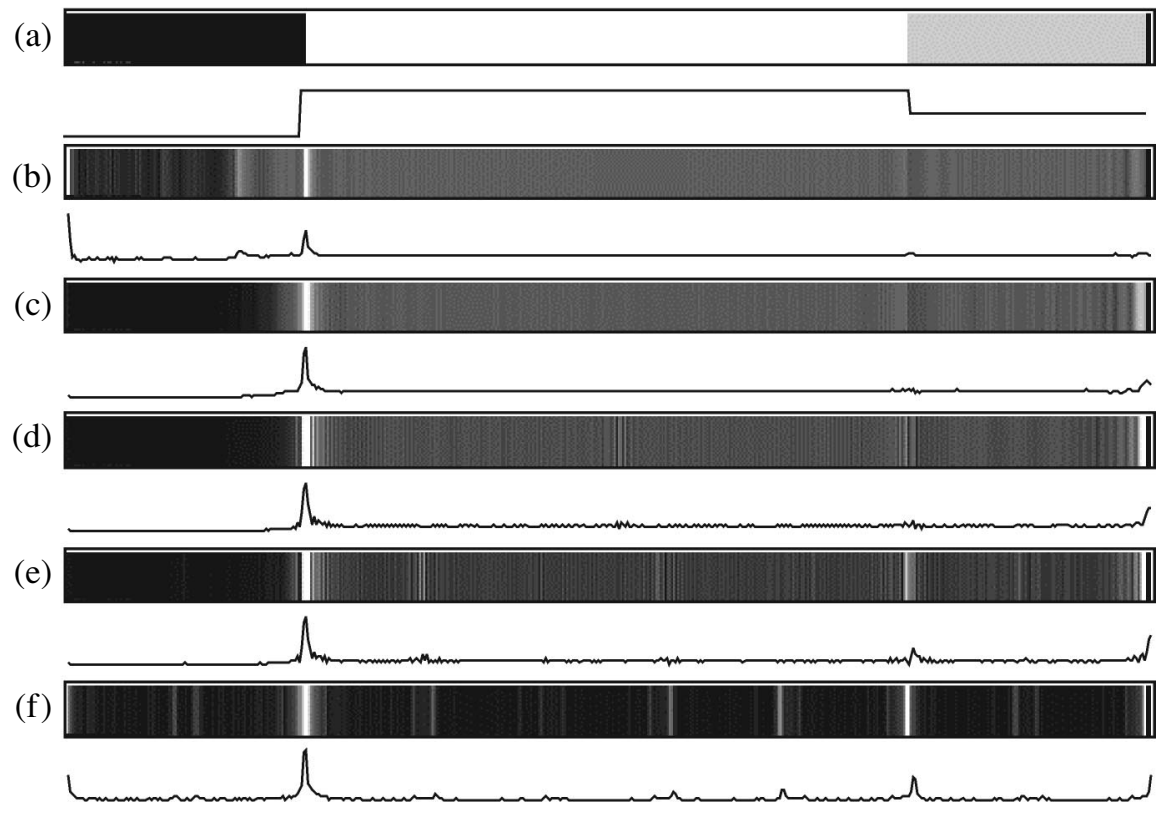

Fig. 2. Reconstruction of perfect edges of different contrasts $(C=1$, left side; $C=0.3$, right side) from the phase of the fractional FT for different $\alpha$ : (a) original image and (below) its line profile; (b-f) image reconstructed from the phase of the fractional FT for $\alpha=$ (b) $\pi / 20$, (c) $3 \pi / 20$, (d) $\pi / 4$, (e) $7 \pi / 20$, and (f) $\pi / 2$.

mation in the different fractional domains. The program for the calculation of the two-dimensional fractional FT is based on the algorithm proposed in [5]. In Figs. 1a and $2 \mathrm{a}$, the image and its line profile corresponding to two perfect edges of different contrasts $C=1$ (left side) and $C=0.3$ (right side) is shown. In Figs. 1b-1f, the images and their line profiles reconstructed from the amplitude-only data in the fractional FT domains for the angles $\alpha=\pi / 20,3 \pi / 20, \pi / 4,7 \pi / 20$, and $\pi / 2$, respectively, are displayed. We observe that edges of different 


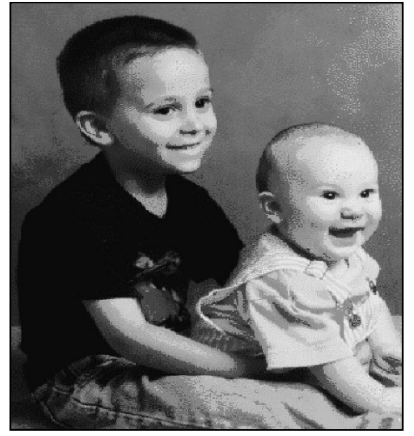

Fig. 3. Original image: photograph of children from the Matlab archive.
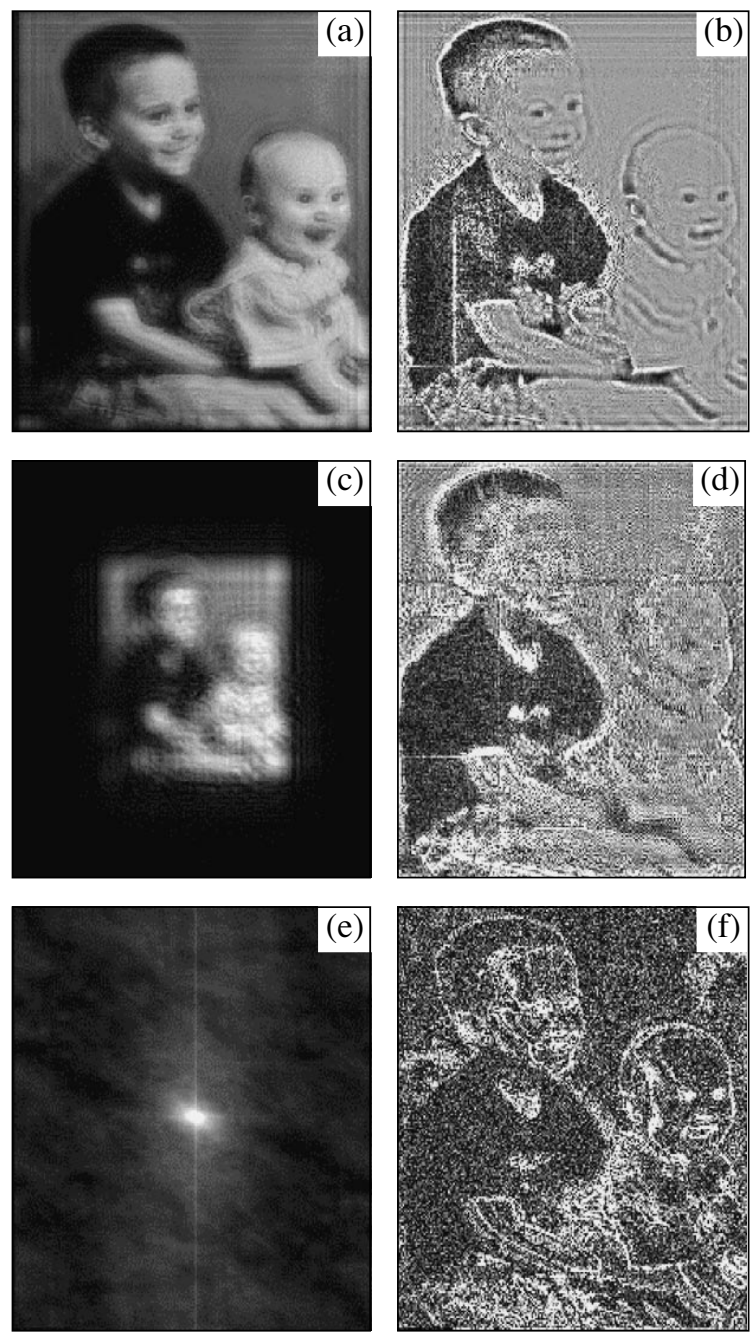

Fig. 4. Images reconstructed from amplitude-only (left side) and phase-only (right side) data in the fractional Fourier domain for different angles $\alpha=(a, b) \pi / 20$; (c, d) $\pi / 4$; $(e, f) \pi / 2$ by applying the inverse fractional FT for the corresponding angles. contrast can be identified from the amplitude-only data in the fractional FT domain for small angles $\alpha \leq \pi / 20$ (Fig. 1b). For larger parameters $\alpha$, due to dependence of the reconstruction procedure on the shift, the image is progressively squeezed with respect to the original one (Figs. 1c-1e) and becomes symmetrical for the Fourier domain $\alpha=\pi / 2$ (Fig. 1f), being the inverse Fourier transform of a real function.

In Figs. 2a-2f, the images of two perfect edges reconstructed from the phase-only data in the different fractional FT domains (original image, $\alpha=\pi / 20, \alpha=$ $3 \pi / 20, \alpha=\pi / 4, \alpha=7 \pi / 20$, and $\alpha=\pi / 2$, respectively) are shown. One can see that, for $\alpha \leq \pi / 4$ (Figs. 2b-2d), the location of the high-contrast edge is well defined. For bigger values of the parameter $\alpha$, both edges are observed (Figs. 2e, 2f). At the same time, for these values of $\alpha$, additional peaks in the intensity of the reconstructed image appear. Therefore, the right choice of the intensity threshold is important for edge detection based on reconstruction from the phase-only information in the fractional FT domain (including the FT domain).

Let us now analyze the reconstruction of a natural image from amplitude-only and phase-only data in the fractional Fourier domain. The original image is a photograph of children taken from the Matlab archives. It contains $400 \times 318$ pixels and is shown in Fig. 3 .

In Fig. 4, images reconstructed from the amplitude only (left side) and phase only (right side) of the fractional FT for angles $\alpha=\pi / 20$ (Figs. 4a, 4b), $\alpha=\pi / 4$ (Figs. 4c, 4d), and $\alpha=\pi / 2$ (Figs. 4e, 4f) are displayed. We observe that, for small angles $\alpha \approx \pi / 20$, the significant information about the original image can be obtained from the amplitude-only (Fig. 4a) as well as the phase-only (Fig. 4b) data. Note that the image reconstructed from the amplitude of the fractional FT is slightly shifted toward the center with respect to the original image, while the image localization is preserved for reconstruction from phase-only data in the fractional domain. Comparing, for example, the images of the children in Figs. $4 a$ and $4 b$, we notice that the reconstruction from the phase data is better for highcontrast details, while low-contrast objects, such as the baby's face, are better reconstructed from the amplitude. Increasing the angle of the fractional FT, we observe that the reconstruction from the amplitude data rapidly loses information about the original image. Thus, in Fig. 4c, $(\alpha=\pi / 4)$, only the outline of the children's figures can be recognized. The image reconstructed from the phase data still contains significant information about the original image. Although some details can not be distinguished, the faces and arms of the children are still recognizable (Fig. 4d).

The amplitude of the FT does not permit any significant level of detail of the original image to be revealed (Fig. 4e). In the meantime, the image reconstructed from the FT phase (Fig. 4f) clearly shows the edges of the original image. The reconstruction from the phase 
in the Fourier domain eliminates the information about the intensity levels of the original image and almost binarizes it. We observe only the edges of the original image on the noisy background.

It has been shown that the image reconstructed from the amplitude of the fractional FT reveals the main features of the original signal only for relatively small angular parameters. Therefore, amplitude-only filters can be applied for pattern recognition tasks in the fractional Fourier domain only for a limited region of the parameter $\alpha$.

Based on the phase information in the fractional FT domains, significant details of the image can be obtained for almost all angles. This means that phaseonly filters applied in the fractional Fourier domain can be used for optical image processing. The quality of image reconstruction from the phase-only data in the fractional Fourier domain depends on the image contrast and the parameter $\alpha$.

\section{ACKNOWLEDGMENTS}

This work was supported by grant no. TIC20002791E from the Spanish Ministry of Science and Technology.

\section{REFERENCES}

1. A. Kozma and D. Kelly, Appl. Opt. 4, 387 (1965).

2. A. V. Oppenheim and J. S. Lim, Proc. IEEE 69, 529 (1981).

3. J. R. Fienup, in International Trends in Optics, Ed. by J. W. Goodman (Academic, Ann Arbor, Mich., 1991), Vol. 1.

4. A. W. Lohmann, D. Mendlovic, and G. Shabtay, J. Opt. Soc. Am. A 14, 2901 (1997).

5. H. M. Ozaktas, Z. Zalevsky, and M. A. Kutay, The Fractional Fourier Transform with Applications in Optics and Signal Processing (Wiley, New York, 2001) (see refs. in).

6. A. W. Lohmann, J. Opt. Soc. Am. A 10, 2181 (1993).

7. D. Mendlovic, H. M. Ozaktas, and A. W. Lohmann, Appl. Opt. 34, 303 (1995). 